\title{
Free space operating microwave imaging device for bone lesion detection: a phantom investigation
}

\author{
Banafsheh Khalesi, Behnaz Sohani, Navid Ghavami, Mohammad Ghavami, Sandra Dudley, Gianluigi Tiberi
}

\begin{abstract}
In this letter, a phantom validation of a low complexity microwave imaging device operating in free space in the 1-6.5 GHz frequency band is presented. The device, initially constructed for breast cancer detection, measures the scattered signals in a multi-bistatic fashion and employs an imaging procedure based on Huygens principle. Detection has been achieved in both bone fracture lesion and bone marrow lesion scenarios using the superimposition of five doublet transmitting positions, after applying the rotation subtraction artefact removal method. A resolution of $5 \mathrm{~mm}$ and a signal to clutter ratio $(3.35$ in linear scale) are achieved confirming the advantage of employing multiple transmitting positions on increased detection capability.
\end{abstract}

Index Terms-Bone lesion, The Huygens, Microwave imaging.

\section{INTRODUCTION}

A MONG the various imaging techniques, microwave imaging is accepted as an attractive technique for diagnostic applications due to its capabilites and safety to produce images of human organs [1]. Microwave imaging can be used for detection and monitoring of a variety of diseases since there is a contrast at microwave frequencies between the dielectric properties of healthy tissues and tissues with lesions [2]. Microwave imaging techniques are based on the scattering of electromagnetic waves. Specifically, a lesion immersed in a healthy tissue when illuminated by an electromagnetic field, transmitted by a dedicated antenna may generate a scattered field, which, if appropriately measured and processed, can be used to detect and locate the lesion itself.

Wide ranging research has been performed to build and develop scanning systems using microwave imaging techniques suitable for medical applications. Many microwave imaging devices have been designed for breast lesion [3], [4] and brain stroke [5]- [7] detection. Recent applications have been proposed for bone imaging: for instance, the authors in [8] designed a microwave scanning system which consists of an antenna array immersed in a matching liquid. Bone lesion detection through microwave imaging has also been investigated via phantom measurements in an anechoic chamber, using two antennas in free space employing an imaging procedure based on Huygens principle [9].

This project and the project leading to this application have received funding from the European Union's Horizon 2020 research and innovation programme under the Marie Sklodowska-Curie grant agreement No. 872752. and No. 793449, respectively.

B. Khalesi, B. Sohani, M. Ghavami, S. Dudley and G. Tiberi are with the School of Engineering, London South Bank University, London SE1 0AA

,U.K.;(khalesib@1sbu.ac.uk; ghavamim@1sbu.ac.uk; dudleyms@1sbu.ac.uk; g.tiberi@iet.unipi.it)

N. Ghavami is with UBT-Umbria Bioengineering Technologies, Assisi 06081, Italy; (navgh1988@yahoo.co.uk.)
Recently, a portable microwave imaging device, operating in free space with two azimuthally-rotating antennas has been constructed and used for breast cancer detection [10]. Specifically, the two antennas rotate around the breast to collect the signals in a multi-bistatic fashion. The purpose of this letter is to investigate and quantify, through phantom experiments, the capability of this imaging device to detect bone lesions. In this context, multilayered phantoms mimicking bone fractures or bone marrow lesions are realized using millimetric, cylindrically shaped inclusions to emulate lesions. An artefact removal procedure has been completed using a rotation subtraction method [9] comprising performing imaging after subtracting two measurements collected using two slightly displaced transmitting positions. Subsequently, a rigorous image quantification procedure has been implemented to assess the detection capability in two scenarios, i.e., bone fracture and bone marrow lesion. Finally, to evaluate detection capability in a more realistic scenario, the measurement was repeated by the cylindrically-shaped inclusion with an inclusion having a high-eccentric elliptical cross-section, i.e. flatshaped. It is worthwhile to point out that the capability of Huygens principle-based microwave imaging to detect bone lesions was first demonstrated through phantom measurements inside an anechoic chamber [9]; here instead we performed a phantom investigation using a microwave imaging device (named MammoWave, shown in Fig. 1(a)) [10].

\section{EXPERIMENTAL CONFIGURATION}

\section{A. Microwave imaging device description}

The microwave imaging device entails an aluminum cylindrical hub containing two antennas, one transmitting (tx) and one receiving (rx). The hub is internally covered by microwave absorbers, and is equipped with a hole and a cup, allowing the insertion of the object to be imaged. The antennas are installed at the same height, in free space and can rotate around the azimuth to collect microwave signals from different angular positions (as shown in Fig. 1(b)). More details of the device can be found in [10]. The tx and $\mathrm{rx}$ are connected to a 2port VNA (S5065, Copper Mountain, Indianapolis, IN) which operates up to $6.5 \mathrm{GHz}$. Measurements have been performed by recording the complex S21 in a multi-bistatic fashion, i.e. for each transmitting position $\mathrm{tx}_{m}$ the receiving antenna is moved to measure the received signal every $4.5^{\circ}$, leading to a total of 80 receiving points $\mathrm{rx}_{n p}$. Concerning the transmitting positions, all experiments have been performed by employing 10 transmitting position, displaced in 5 doublets centered at $0^{\circ}, 72^{\circ}, 144^{\circ}, 216^{\circ}$, and $288^{\circ}$. Fig. 1(c) illustrates the set up configuration. As Fig. 1(c) shows, in each doublet the two 


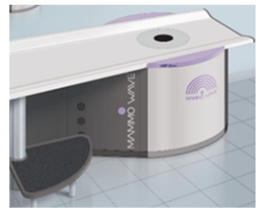

(a)

(b)

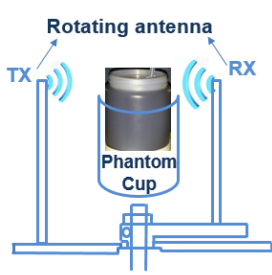

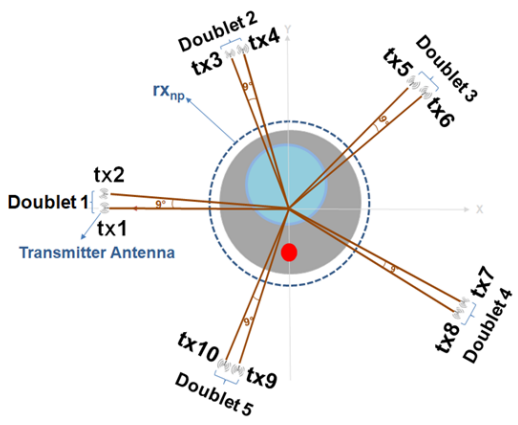

(c)
Fig. 1: In (a) there is the MammoWave microwave imaging device [10], having external diameter of $100 \mathrm{~cm}$. Inside the cylindrical hub there are the tx (Horn-type) and rx (Vivaldi-type) antennas, having Voltage Standing Wave Ratio $<3$ in the band 1-6.5 GHz, (b) Crosssectional diagram of the device and phantom cup, (c) Pictorial topview of the device configuration.

transmitting positions are displaced by $9^{\circ}$. For each tx and rx position, the complex S21 is collected from 1 to $6.5 \mathrm{GHz}$, with $5 \mathrm{MHz}$ sampling. This frequency range can be considered appropriate for bone imaging [8], [9].

\section{B. Phantom descriptions}

Two bone lesion phantoms comprising three layers have been fabricated. The three layers mimic: i) the cortical bone (external layer); ii) the bone marrow layer (internal layer); and iii) a lesion. In the first phantom, which represents a bone fracture, the lesion has been placed between the external and internal layers, as shown in Fig. 2(a). In the second phantom (Fig. 2(b)), representing a bone marrow lesion, the lesion has been placed inside the internal layer. Dedicated liquids have been purchased from the ZMT Zurich MedTech Company [11] to mimic the different bone layers. The TLe11.5C.045 liquid oil has been employed to represent the external layer, which mimics the cortical bone layer. The internal layer, imitating the bone marrow layer, has been fabricated using Tle $5 \mathrm{C} 24$ liquid oil. The lesion has been mimicked through a combination of $40 \%$ Glycerol and $60 \%$ water [12]. The permittivity and conductivity values for TLe11.5C.045, Tle5C24, and the combination of $40 \%$ Glycerol and $60 \%$ water are: $\varepsilon_{r}=7$ and $\sigma=0.3 \mathrm{~S} / \mathrm{m} ; \varepsilon_{r}=5$ and $\sigma=0.2 \mathrm{~S} / \mathrm{m} ; \varepsilon_{r}=60$ and $\sigma=2 \mathrm{~S} / \mathrm{m}$, respectively. It should be highlighted that the permittivity and conductivity values are calculated at $2 \mathrm{GHz}$ and such dielectric properties can be considered representative of cortical bone, bone marrow and a lesion constituted of blood [9].

Two cylindrically shaped plastic containers with diameters of $11 \mathrm{~cm}$ and $7 \mathrm{~cm}$ have been used to maintain the bone cortical and bone marrow equivalent materials, respectively. A cylindrically shaped tube of $4 \mathrm{~mm}$ diameter has been employed to contain an inclusion (see Fig. 2). The phantoms have been placed inside the cup of the microwave imaging device as shown in Fig. 1(b); in more details, the bone marrow lesion phantom has been positioned as shown in Fig. 3(a) while the bone fracture lesion phantom has been located as shown in Fig. 3(b). The cortical bone layer, bone marrow layer and

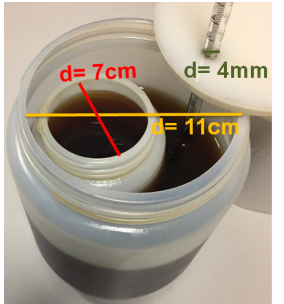

(a)

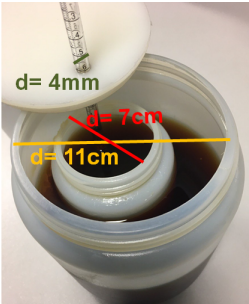

(b)

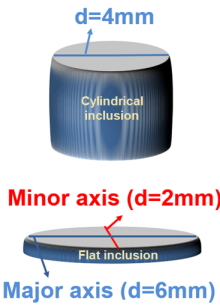

(c)
Fig. 2: Fabricated phantoms for (a) bone fracture, (b) bone marrow lesion, and (c) cross-section of cylindrical inclusion compared to the flat inclusion.

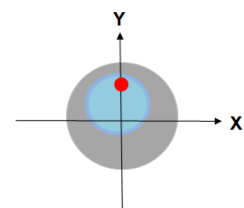

(a)

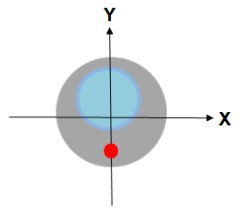

(b)
Fig. 3: Schematic view of bone marrow (a), and bone fracture lesion (b).

inclusion are presented in gray, light blue and red, respectively. Additionally, two further scenarios have been considered, slightly modifying the bone marrow lesion phantom. First, the cylindrically-shaped inclusion was replaced with an inclusion having a high-eccentric elliptical cross-section, i.e. flat-shaped (major axis $6 \mathrm{~mm}$, minor axis $2 \mathrm{~mm}$ ). Next, the TLe11.5C.045 was substituted with a combination of $90 \%$ Glycerol and $10 \%$ water, having $\varepsilon_{r}=16$ and $\sigma=1.1 \mathrm{~S} / \mathrm{m}$ at $2 \mathrm{GHz}$ [12]; such dielectric properties can be considered representative of a muscle and fat tissue [13].

\section{Imaging procedure}

The measured complex S21 have been processed through an imaging algorithm based on Huygens principle [14] to generate the images. Assuming that rx can be rotatably moved to measure the received signal at the points $\operatorname{rx}_{n p} \equiv\left(a_{0}, \phi_{n p}\right) \equiv$ $\vec{\rho}_{n p}$ displaced along a circular surface having radius $a_{0}$, the received signals can be expressed as $\mathrm{S}_{21} 1_{n, m}\left(a_{0}, \phi_{n p} ; \mathrm{tx}_{m} ; f\right)$ with $n p=1,2, \ldots, 80, m=1,2, \ldots, 10$, and $f$ representing the frequency. The rotation subtraction strategy has been implemented for artefact removal by employing (1). This procedure has been performed through subtraction between transmitting position $m$ and transmitting position $m+1$, with $m$ and $m+1$ belonging to the same doublet:

$$
\begin{aligned}
& E_{\mathrm{HP}, 2 \mathrm{D}}^{\mathrm{rcstr}}\left(\rho, \phi ; \mathrm{tx}_{m}-\mathrm{tx}_{m+1} ; f\right) \\
& \propto \Delta s \sum_{n p=1}^{N_{P T}}\left(\left(\mathrm{~S} 21_{n p, \mathrm{tx}_{m}}^{\mathrm{known}_{\mathrm{k}}}-\mathrm{S} 21_{n p, \mathrm{tx}_{m+1}}^{\mathrm{known}_{m}}\right) G\left(k_{1}\left|\overrightarrow{\rho_{n p}}-\vec{\rho}\right|\right)\right)
\end{aligned}
$$

where $(\rho, \phi) \equiv \vec{\rho}$ is the observation point, $\Delta s$ is the spatial sampling and $N_{P T}$ is the number of receiving points. The component $k_{1}$ indicates the wave number, and $G$ is the Green's function. The "reconstructed" internal field is indicated by the string "rcstr" while the string HP indicates that the Huygens based procedure will be employed in (1). More details can be found in [14], [15]. The intensity of the final images is given by the summation of different images corresponding 
to different transmitting doublets, each one obtained through the non-coherent summation of all frequency contributions. Therefore, the combined image of 5 transmitting position doublets has been generated through the following equation ( $N_{F}$ being the number of frequency samples):

$$
\begin{aligned}
I(\rho, \phi) & =\sum_{m=1}^{5} I\left(\rho, \phi ; \mathrm{tx}_{2 m-1}-\mathrm{tx}_{2 m}\right) \\
& =\sum_{m=1}^{5} \sum_{i=1}^{N_{F}}\left|E_{\mathrm{HP}, 2 \mathrm{D}}^{\mathrm{rcstr}}\left(\rho, \phi ; \mathrm{tx}_{2 m-1}-\mathrm{tx}_{2 m} ; f_{i}\right)\right|^{2}
\end{aligned}
$$

\section{Imaging quantification}

To quantify the algorithm's detection capabilities, two metrics have been introduced and calculated: i) resolution and ii) signal to clutter ratio $(\mathrm{S} / \mathrm{C})$. Specifically, the resolution is defined as a dimension of the region whose normalized intensity is above 0.5 [15]; $\mathrm{S} / \mathrm{C}$ was defined as the ratio between maximum intensity evaluated in the region of the lesion divided by the maximum intensity outside the region of the lesion [16].

\section{EXPERIMENTAL RESULTS}

Experimentations have been executed by considering both the 5 individual transmitting position doublets, and the combination of 5 transmitting position doublets. All the obtained microwave images have been gathered in Fig. 4 and Fig. 5, exhibiting bone fracture lesion and bone marrow lesion, respectively. The images are obtained after employing the rotation subtraction between two doublet positions, functioning as an artefact removal procedure. The red arrows in the figures indicate the true location of the inclusion.

For both bone fracture lesion and bone marrow lesion, the images in Figs. 4(a) to 4(e) and 5(a) to 5(e) represent the results of employing individual doublets, whilst Figs. 4(f) and 5(f) represent the result of employing the combination of five transmitting position doublets, i.e. applying (2).

Imaging performance has been investigated through image quantification. For this purpose, in order to evaluate the impact of transmitting positions in achieving detection, resolution and $\mathrm{S} / \mathrm{C}$ have been calculated for the obtained images of five individual transmitting position doublets and the combination of 5 transmitting position doublets. Results have been collected and summarised in Table I for both bone fracture and bone marrow lesions. Figs. 6(a) and 6(b) show the images obtained employing one single sample frequency, i.e., a single frequency contribution calculated for the central frequency $(3.75 \mathrm{GHz})$, for bone fracture lesion and bone marrow lesion, respectively. Finally, Figs. 7 (a) and 7(b) show the images corresponding to the combination of 5 transmitting position doublets obtained for the bone marrow lesion phantom when using a flatshaped inclusion and after replacing the TLe11.5C.045 with a combination of $90 \%$ Glycerol and $10 \%$ water, respectively. Both images are the combination of 5 transmitting position doublets.

\section{Discusion}

In order to investigate the ability of the proposed microwave imaging device to detect bone lesions, experiments have been

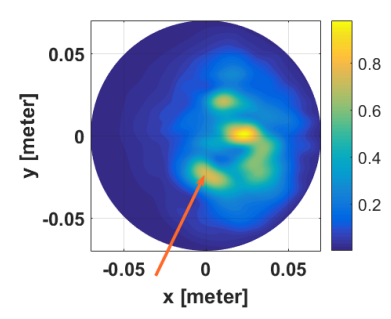

(a)

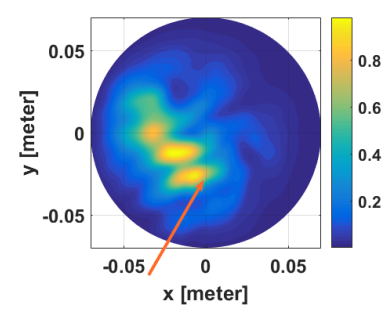

(c)

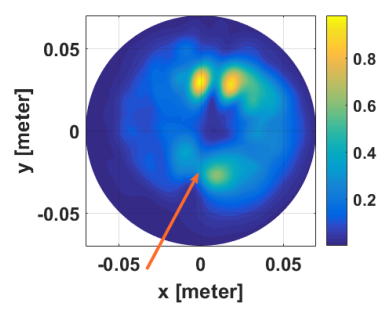

(e)

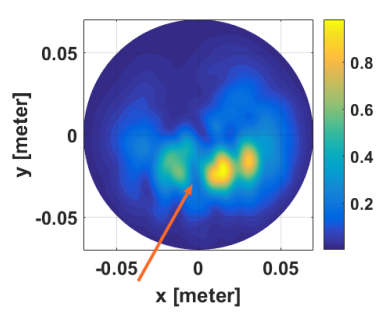

(b)

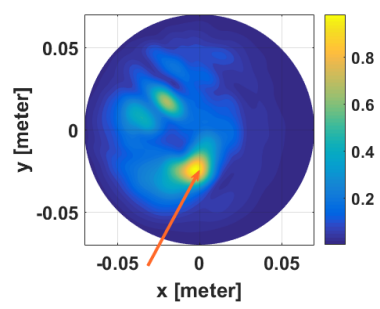

(d)

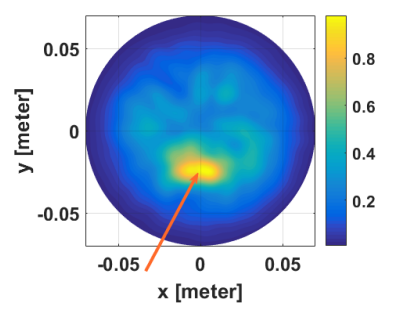

(f)
Fig. 4: Microwave images of bone fracture lesion employing frequency 1-6.5 GHz. (a) to (f) represent the resulting images for first, second, third, fourth, fifth doublets and combination of 5 transmitting position doublets, respectively.

TABLE I: Resolution ( $\mathrm{mm}$ ) and S/C for bone lesion.

\begin{tabular}{|c|c|c|c|c|}
\hline & \multicolumn{2}{|c|}{ Bone fracture } & \multicolumn{2}{c|}{ Bone Marrow } \\
\hline No. doublet & Resolution & S/C & Resolution & S/C \\
\hline First doublet & N/A & $<1$ & 7 & 1.8132 \\
Second doublet & 6 & 1.1585 & 6 & 1.5795 \\
Third doublet & 9 & 1.1822 & 6 & 2.5833 \\
Fourth doublet & 6 & 1.4751 & 6 & 1.5316 \\
Fifth doublet & N/A & $<1$ & 6 & 1.9960 \\
Combining 5 doublets & 5 & 2.2650 & 5 & 3.3512 \\
\hline
\end{tabular}

performed using realistic phantoms with a very thin inclusion (diameter $4 \mathrm{~mm}$ ), employing both individual transmitting positions doublets and their combination.

Rotation subtraction artefact removal has been employed to suppress artefacts, i.e. the image of the transmitter and the reflections of the layers. However, it may happen that even beyond artefact removal, residual clutter may mask the inclusion. Residual clutter is due to the imperfect cancellation of the transmitting antenna, inappropriate cancellation of the first layer reflection or can be due to multiple reflections occurring inside the phantom that cannot be cancelled completely.

For the bone fracture lesion, detection is achieved using the second transmitting position doublet (Fig. 4(b)), third doublet (Fig. 4(c)) and fourth doublet (Fig. 4(d)). However, detection is 


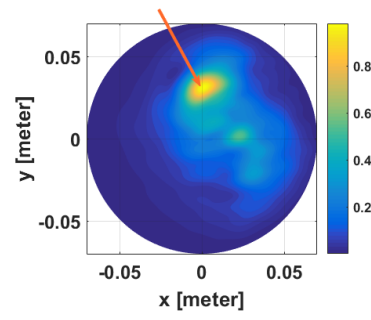

(a)

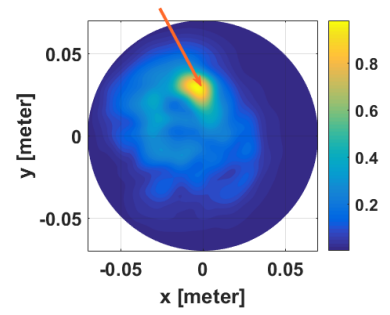

(c)

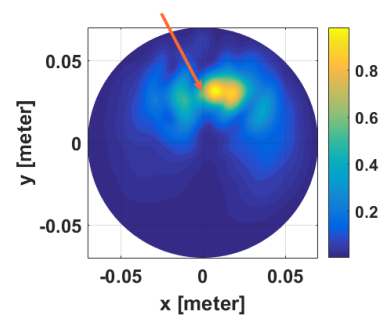

(e)

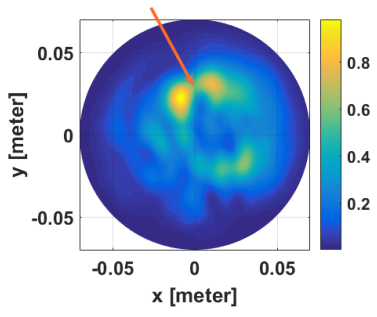

(b)

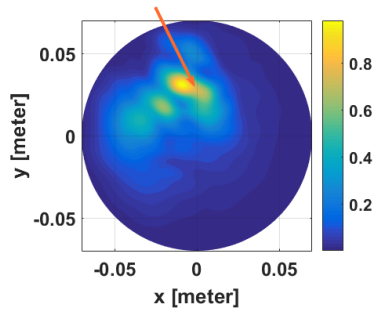

(d)

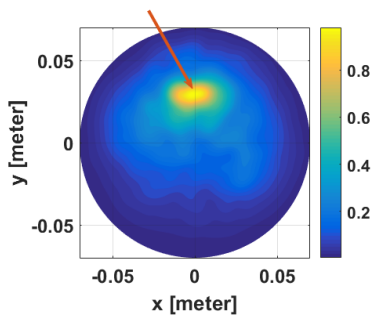

(f)
Fig. 5: Microwave images of bone marrow lesion employing frequency 1-6.5 GHz. (a) to (f) represent the resulting images for first, second, third, fourth, fifth doublets and combination of 5 transmitting position doublets, respectively.

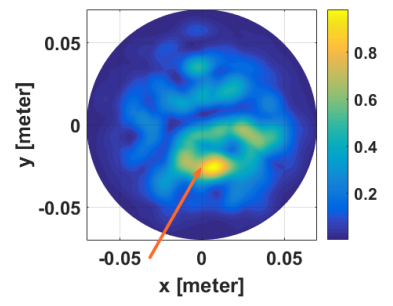

(a)

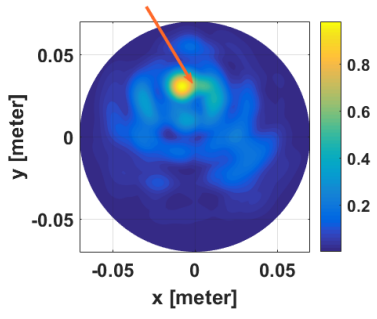

(b)
Fig. 6: Microwave images employing a central frequency $3.75 \mathrm{GHz}$ for (a) bone fracture lesion, (b) bone marrow lesion.

not achieved using the first doublet (Fig. 4(a)) and fifth doublet (Fig. 4(e)), most likely due to residual clutter. Detection is also achieved using the combination of 5 doublets (Fig. 4(f)). For the bone marrow lesion case, as shown in Fig. 5, detection has been successfully achieved in all individual transmitting position doublets and also using the combination of the five doublets.

Concerning image quantification (Table I), in bone fracture lesion the S/C varies up to 1.47 by employing the individual doublets, while in the bone marrow lesion scenario S/C varies from 1.53 to 2.58 using the individual doublets. These values are in good agreement with those from the anechoic cham-

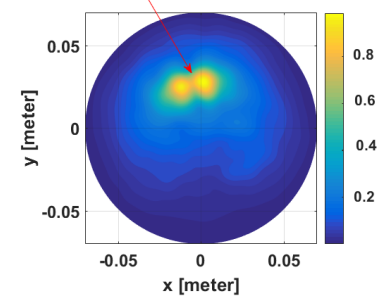

(a)

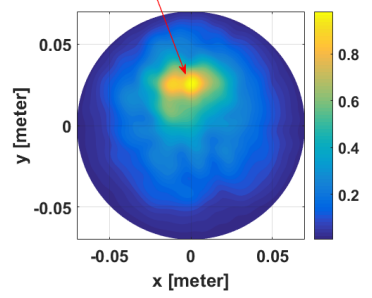

(b)
Fig. 7: Microwave images of bone marrow: (a) using flat inclusion, (b) replacing the TLe11.5C.045 with a combination of $90 \%$ Glycerol and $10 \%$ water, mimicking muscle and fat; $\mathrm{x}$ and $\mathrm{y}$ axes are in meters.

ber measurements [9], where a transmitting position doublet displaced at $5^{\circ}$ or $10^{\circ}$ was employed. Furthermore, Table I shows that, when using the combination of 5 doublets, S/C increases up to 2.26 in bone fracture lesion and 3.35 in bone marrow lesion. It follows that the combination of 5 doublets is beneficial in term of $\mathrm{S} / \mathrm{C}$, a finding which is in agreement with previous studies [14]- [17].

The higher values of $\mathrm{S} / \mathrm{C}$ for bone marrow lesion with respect to the bone fracture may be related to the existence of more residual clutter in the fracture lesion scenario, as the fracture lesion scenario is more asymmetric. This is confirmed from Fig. 6, which refers to one single frequency contribution.

Concerning the resolution, according to the collected data in Table I, using the combination of 5 doublets for both bone fracture and bone marrow lesion, the best resolution value equal to $5 \mathrm{~mm}$ was achieved. This is in excellent agreement with the optical resolution limit of $\lambda_{1, f_{\max }} / 4$, where $\lambda_{1, f_{\max }}$ represents the wavelength when considering a dielectric constant equal to the arithmetical average of the two layers calculated at the highest frequency of $6.5 \mathrm{GHz}$ [15], [16]. Fig. 7(a) shows detection of the flat-shaped inclusion; interestingly, two spots appear in correspondence of the major axis extremities. This might suggests that lesions of $2 \mathrm{~mm}$ could be detected, even if, in the images, they might appear, larger in size. Fig. 7(b) shows detection also when a muscle and fat mimicking layer is employed as an external layer. Fig. 7 confirms that detection can be achieved in more realistic phantoms, even if a S/C lower than that of Fig. 5(f) can be (visually) appreciated. However, a limitation of the investigation presented in this letter is that phantoms having a cylindrically shaped external layer were always used. Next steps will be focused on the construction and use of anthropomorphic phantoms.

\section{CONClusion}

The capability of HP-based microwave imaging to detect bone lesions was first demonstrated through phantom measurements in an anechoic chamber [9]. Here, a phantom investigation using a microwave imaging device (based on HP) for bone lesions detection has been performed. Resolution of $5 \mathrm{~mm}$ and the $\mathrm{S} / \mathrm{C}$ of 3.35 have been obtained by employing the combination of 5 transmitting position doublets in the imaging procedure and using a frequency band of 1-6.5 GHz. The microwave imaging device is safe (no X-rays), portable and it has low complexity since it employs only two rotating antennas operating in free space coupled through a VNA. 


\section{REFERENCES}

[1] 1. E. Larsen and J. H. Jacobi, "Microwave scattering parameter imagery of an isolated canine kidney," Med phys., vol. 6, no. 5, pp. 394-403, Sep-Oct 1979.

[2] M. Lazebnik et al., "A large-scale study of the ultrawideband microwave dielectric properties of normal, benign and malignant breast tissues obtained from cancer surgeries," Physics in Medicine and Biology., vol. 52, no. 20, pp. 6093-6115, Oct. 2007.

[3] E. Porter, M. Coates, and M. Popovic, "An early clinical study of timedomain microwave radar for breast health monitoring," IEEE Trans. Biomed. Eng., vol. 63, no. 3, pp. 530-9, Mar 2016.

[4] L. Wang, "Multi-Frequency Holographic Microwave Imaging for Breast Lesion Detection,” IEEE Access., vol. 7, pp. 83984-83993, Jun. 2019.

[5] S. Y. Semenov and D. R. Corfield, "Microwave tomography for brain imaging: Feasibility assessment for stroke detection," Int. J. Antenna Propag,, vol. 2008, pp. 1-8, 2008.

[6] V. L. Coli et al., "Detection of Simulated Brain Strokes Using Microwave Tomography," in IEEE Journal of Electromagnetics, RF and Microwaves in Medicine and Biology, vol. 3, no. 4, pp. 254-260, 2019.

[7] A. E. Stancombe, K. S. Bialkowski, and A. M. Abbosh, "Portable Microwave Head Imaging System Using Software-Defined Radio and Switching Network," in IEEE Journal of Electromagnetics, RF and Microwaves in Medicine and Biology, vol. 3, no. 4, pp. 284-291, 2019.

[8] G. Ruvio et al., "Microwave bone imaging: A preliminary scanning system for proof-of-concept," IET Healthc. Technol. Lett, vol. 3, no. 3, pp. 218-221, Sep 2016.

[9] B. Khalesi et al., "A Phantom Investigation to Quantify Huygens Principle Based Microwave Imaging for Bone Lesion Detection,' Electronics, vol. 8, no. 12, 2019.

[10] A. Vispa et al., "UWB Device for Breast Microwave Imaging: Phantom and Clinical Validations," Measurement., vol. 146, pp. 582-589, Jun. 2019.

[11] Zurich Med Tech. Available: https://zmt.swiss/validation-hw/tsm/tle5c-24-2450/ [Accessed: 26-102019].

[12] P.M. Meaney et al., "Characterization of Glycerin: Water Mixtures: Implications for Use as a Coupling Medium in Microwave Tomography,' IEEE Transactions on Microwave Theory and Techniques, vol. 65, no.5, pp. 1471-1478, 2017.

[13] C. Gabriel et al., "The dielectric properties of biological tissues: I. literature survey," Physics in Medicine and Biology, vol. 41, no. 11, pp. 2231-2249,1996.

[14] G. Tiberi et al., "Ultrawideband microwave imaging of cylindrical objects with inclusions," IET Microw. Antennas Propag., vol. 5, vol. 5 , no. $12,2011$.

[15] N. Ghavami et al., "UWB Microwave Imaging of Objects with Canonical Shape," IEEE Trans. Antennas Propag., vol. 60, no. 1, pp. 231-239, Jan. 2012.

[16] E. C. Fear et al., "Confocal microwave imaging for breast cancer detection: Localization of tumors in three dimensions," IEEE Trans. Biomed. Eng., vol. 49, no. 8, pp. 812-822, Aug. 2002.

[17] L. Sani et al., "Novel microwave apparatus for breast lesions detection: Preliminary clinical results," Biomed. Signal Process. Control., vol. 52, pp. 257-263, July. 2019. 